\title{
复 \\ Xica - a história de vida do peixe-boi-marinho (Trichechus manatus) mais velho do Brasil: relato de caso
}

\author{
[Xica - the life story of the oldest Antillean manatee (Trichechus manatus) in Brazil: Case report]
}

\section{"Relato de Caso/Case Report"}

\author{
Fábia de Oliveira Luna $^{1 *}$, Fernanda Loffler Niemeyer Attademo ${ }^{1,2,3,4}$
}

\author{
${ }^{1}$ Centro Nacional de Pesquisa e Conservação de Mamíferos Aquáticos, Instituto Chico Mendes de Conservação da \\ Biodiversidade - ICMBio, Santos-SP, Brasil. \\ ${ }^{2}$ Projeto Cetáceos da Costa Branca, Universidade do Estado do Rio Grande do Norte, Mossoró-RN, Brasil. \\ ${ }^{3}$ Centro de Estudos e Monitoramento Ambiental - CEMAM, Areia Branca-RN, Brasil. \\ ${ }^{4}$ Instituto Brasileiro para Medicina da Conservação, Recife-PE, Brasil. \\ *Autora para correspondência/Corresponding author: E-mail: fabialunacma@gmail.com
}

\section{Resumo}

O peixe-boi-marinho (Trichechus manatus), um dos mamíferos aquáticos mais ameaçados de extinção do Brasil, tem o resgate e a reabilitação destes animais como importante ferramenta de conservação. Xica foi resgatada de um cativeiro inadequado na praça do Derby, Pernambuco, apresentando problemas clínicos e comportamento estereotipado. A transferência de Xica para o Projeto Peixe-Boi lhe deu melhor qualidade de vida e acompanhamento médico veterinário adequado. Este trabalho teve o objetivo de relatar a trajetória de vida de Xica, destacando a longevidade e as informações clínicas e reprodutivas de relevância durante os cerca de 52 anos que ela viveu. Foram utilizadas as informações de histórico, prontuários clínicos e relatório de necropsia da instituição. Verificou-se que Xica desenvolveu uma escoliose na coluna vertebral e na região lombar, e apresentou uma cicatriz na região dorsal devido a ferimentos causados no cativeiro inadequado. Ela teve quatro parições: dois filhotes vivos e dois natimortos. A escoliose provavelmente era motivo de dores extremas, podendo ter influenciado no comportamento da fêmea. Sem contribuições de prole para populações nativas, Xica deixou uma enorme colaboração para a conservação da espécie provendo informações sobre longevidade, saúde, comportamento e reprodução.

Palavras-chaves: longevidade; escoliose; reprodução; Trichechus manatus.

\begin{abstract}
The West Indian manatee (Trichechus manatus), one of the most endangered aquatic mammals in Brazil, has the rescue and rehabilitation of these animals as an important conservation tool. Xica was rescued from an inappropriate captivity in Praça do Derby (Derby public square), Pernambuco, presenting clinical problems and stereotyped behavior. The transfer of Xica to the Peixe-Boi Project gave her a better quality of life and adequate veterinary medical care. This work aimed to report on Xica's life trajectory, highlighting her longevity and relevant clinical and reproductive information during the approximately 52 years she lived. The institution's history information, clinical records and necropsy report were used. It was found that Xica developed scoliosis in the spine and lumbar region and showed a scar on the dorsal region due to injuries caused by inappropriate captivity. She had four births: two live calves and two stillborns. Scoliosis probably provoked extreme pain and may have influenced her behavior. Without offspring contributions to native populations, Xica left an enormous contribution to the conservation of the species by providing information on longevity, health, behavior, and reproduction.
\end{abstract}

Keywords: longevity; scoliosis; reproduction; Trichechus manatus. 


\section{Introdução}

O peixe-boi-marinho (Trichechus manatus) é classificado como "em perigo de extinção" (EN) no Brasil (ICMBio, 2018). A espécie, historicamente ocorria desde o estado do Espírito Santo, Brasil até o estado da Flórida, nos Estados Unidos. Em decorrência da forte pressão antrópica, a espécie foi extinta no limite sul de ocorrência entre os estados de Sergipe e Espírito Santo (Luna e Passavante, 2010).

A maior ameaça histórica para a espécie foi a caça, que ocorreu desde o período da colonização do Brasil (Luna et al., 2008; Luna et al., 2018) e foi responsável pela redução drástica no tamanho das populações de peixes-boismarinhos do país (Luna et al., 2018). Entretanto, com a publicação de legislações de proteção à fauna e o avanço de trabalhos socioambientais, a captura intencional foi reduzida, com registros de casos isolados no Maranhão (MA), Pará (PA) e Amapá (AP) (Luna et al., 2008; Luna e Passavante, 2010).

$O$ encalhe de filhotes e o aumento da interação antrópica, entre elas a ingestão de lixo e perda de habitat, atualmente têm sido as principais ameaças para a espécie (Attademo et al., 2015; Balensiefer et al., 2016, Luna et al., 2018). Como estratégia de conservação, as instituições da Rede de Encalhes de Mamíferos Aquáticos do Nordeste (REMANE), entre eles o Instituto Chico Mendes de Conservação da Biodiversidade - Centro Nacional de Pesquisa e Conservação de Mamíferos Aquáticos (ICMBio/CMA), realizam o resgate de animais encalhados, com o objetivo de reabilitar e soltar (Balensiefer et al., 2016; Luna et al., 2018).

Peixes-bois que viviam em recintos inadequados foram também resgatados $\mathrm{e}$ transferidos para a base da instituição, onde passaram a ter cuidados adequados (Luna e Passavante, 2010). O Projeto Peixe-Boi, executado pelo ICMBio/CMA, foi criado em 1980 e desde a criação, além das ações já mencionadas, vem realizando políticas públicas e pesquisas voltadas para a conservação da espécie, contribuindo para que a espécie antes considerada como "Criticamente ameaçada de extinção" (CR), passasse para a atual categoria de EN (Luna et al., 2018).

Os peixes-bois resgatados que por razões clínicas ou comportamentais, que não possuíam condições de serem soltos na natureza, passaram a compor o plantel de visitação pública. Durante a visitação, era realizado um forte trabalho de sensibilização para a conservação da espécie (Luna e Passavante, 2010). No Brasil, o Projeto Peixe Boi foi o primeiro no país a atuar com a reabilitação e manutenção do Trichechus manatus em cativeiro. Portanto, a longevidade da espécie no Brasil era desconhecida, sendo baseada apenas em estudos de outros países.

O presente estudo teve como objetivo fazer o primeiro relato da história de vida e clínica do peixe-boi marinho (Trichechus manatus) mais velho registrado no país, a fêmea Xica que ficou conhecida como "o peixe-boi da praça do Derby".

\section{Descrição do caso}

Xica (\#01S0112/07), um peixe-boi-marinho (Trichechus manatus), fêmea, foi resgatada ainda filhote, em 1963 no município de Goiana, estado Pernambuco e levada para uma propriedade particular, por onde permaneceu por sete anos (Rocha, 1971). Em 1970, Xica foi transferida para o município do Recife, sendo mantida em um recinto na Praça do Derby $\left(8^{\circ} 23^{\prime} 25.75^{\prime \prime} S\right.$, $\left.34^{\circ} 53^{\prime} 58.27^{\prime \prime} O\right)$ até 1992 . O recinto possuía 12 metros de diâmetro e cerca de 1,5 metros de profundidade, considerado pequeno para a espécie. Não se tem registro dos tipos e quantidades de alimentos ofertados neste período. Pelas condições do recinto na praça do Derby, Xica não conseguia realizar movimentos básicos, como mergulhar, sendo possível apenas nadar em círculos. Possivelmente, por esta razão, Xica adquiriu grande escoliose na coluna vertebral e na região lombar (Figura 1).

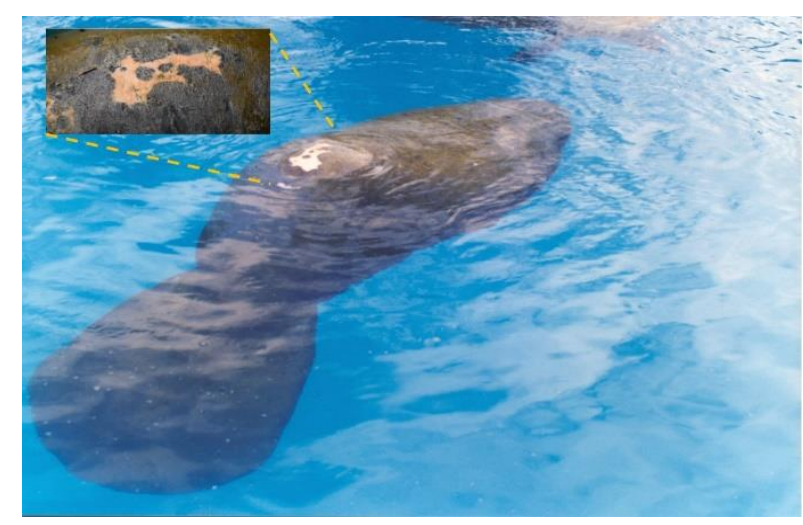

Figura 1. Peixe-boi marinho Xica, apresentando escoliose na coluna vertebral e na região lombar e cicatriz na região dorsal. Foto: Fábia Luna acervo ICMBio/CMA.

Xica também possuía duas cicatrizes esbranquiçadas, ambas na região dorsal, sendo uma cicatriz medindo $21,5 \times 13 \mathrm{~cm}$ outra, mais 
caudal, de 7 x $9 \mathrm{~cm}$, ambas causadas por queimaduras solares ao qual ela era submetida em virtude da pouca profundidade do recinto. Não foram localizados os registros clínicos do período na praça do Derby.

Em fevereiro de 1992, a fêmea foi transferida para Centro de Reabilitação de Animais Silvestres (CRAS) do ICMBio/CMA na base do Projeto Peixe-Boi, localizado na Ilha de Itamaracá, Pernambuco $\quad\left(7^{\circ} 48^{\prime} 33.40^{\prime}\right.$ 'S, $\left.34^{\circ} 50^{\prime} 16.36^{\prime \prime} \mathrm{O}\right)$, onde permaneceu até o óbito. Após a chegada de Xica ao ICMBio/CMA, as informações foram obtidas no banco de dados e prontuários clínicos da instituição.

Ao chegar no ICMBio/CMA, Xica permaneceu com outros indivíduos da mesma espécie, em dois oceanários interligados $(10 \mathrm{~m}$ de diâmetro x 4,40m profundidade, com volume de água de $348,80 \mathrm{~m}^{3}$ cada). Posteriormente, foi construído um terceiro recinto com $174,40 \mathrm{~m}^{3}$ de água ( $10 \mathrm{~m}$ de diâmetro $\mathrm{x} 2 \mathrm{~m}$ profundidade), interligado aos dois primeiros, totalizando $872 \mathrm{~m}^{3}$ de água para os animais (Figura 2). As coletas de amostras biológicas e comportamentais, foram realizadas por meio da licença SISBIO $\mathrm{N}^{\circ} 20685$.

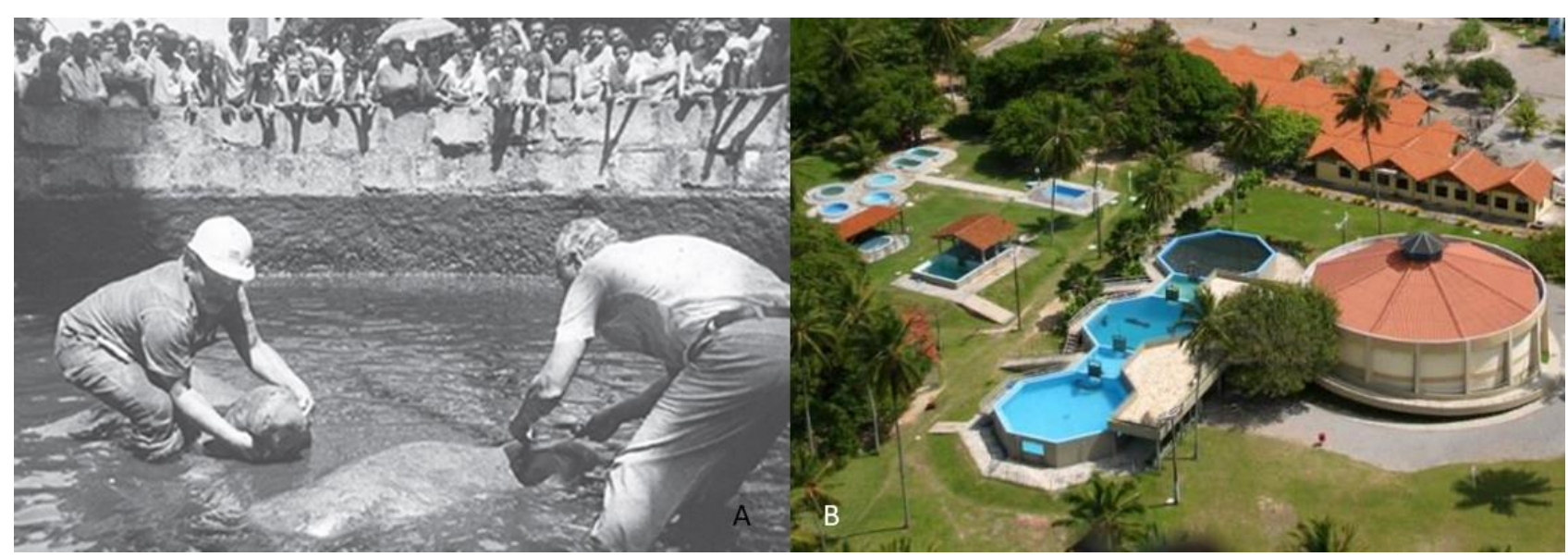

Figura 2. Recintos que o peixe-boi marinho Xica viveu em Pernambuco. A: Recinto na praça do Derby, onde Xica permaneceu de 1970 até 1992, com tamanho pequeno e raso para a espécie. Fonte: Acervo Diário de Pernambuco. B: Três recintos interligados do ICMBio/CMA, onde Xica permaneceu de 1992 até 2015 (Óbito). Fonte: Acervo ICMBio/CMA.

Nos oceanários de Itamaracá, Xica permaneceu junto com fêmeas e machos, mas sempre apresentou comportamento mais arredio que os demais, não permitindo interações interespecífica e permanecendo boa parte do tempo no fundo, praticamente subindo apenas para respirar.

Para a análise biométrica foram consideradas apenas as últimas medidas obtidas em cada ano (1992-2015). As medidas de comprimento total (CT) e de circunferência máxima do tórax (CircT) foram obtidas usando fitas métricas flexíveis, graduadas em centímetros. $\mathrm{O}$ peso, em quilos, foi obtido por meio da equação $\left[\right.$ Peso $=$ CT $\times$ CircT $\left.^{2} \times 0,00003778\right]$. As análises estatísticas foram realizadas usando o software BioEstat 5.3, e as médias foram comparadas pelo teste $\mathrm{t}$ de Student com um nível de significância de 0,05 .

Xica apresentou um ganho de peso de 473 $\mathrm{kg}$, com uma média de ganho de $34 \mathrm{~kg} / \mathrm{ano}$. No período de 1999 até 2003, apresentou perda de
$217 \mathrm{~kg}$ de peso, os quais foram recuperados nos anos seguintes, tendo atingido em 2015 o peso de $825 \mathrm{~kg}$ (Figuras 3 e 4 ).

O último exame hematológico de Xica, antes do óbito, ocorreu em 16.03.2015, cerca de dois meses antes do óbito. Nos exames, não foram verificadas alterações nos exames de hemograma e bioquímico (Harvey et al., 2007; Mendonça et al., 2020).

Para a dieta alimentar, eram calculados entre 5-10\% do peso vivo da fêmea, com itens que variavam conforme o acesso da instituição aos itens e a sazonalidade de cada alimento fornecido. Até 2007, os principais itens ofertados foram capim agulha (Halodule wrightii), algas marinhas (Gracilaria domingensis, Sargassum sp. e outras, conforme disponibilidade), cenoura e alface. Entre 2007 e 2010, com uma drástica redução da abundância de capim agulha na área de colheita, foi reduzida a oferta de capim agulha e algas marinhas e acrescido alface, acelga, cenoura, beterraba, pepino, abóbora e maçã. A partir de 
2010, foram mantidos apenas as verduras, legumes e frutas.

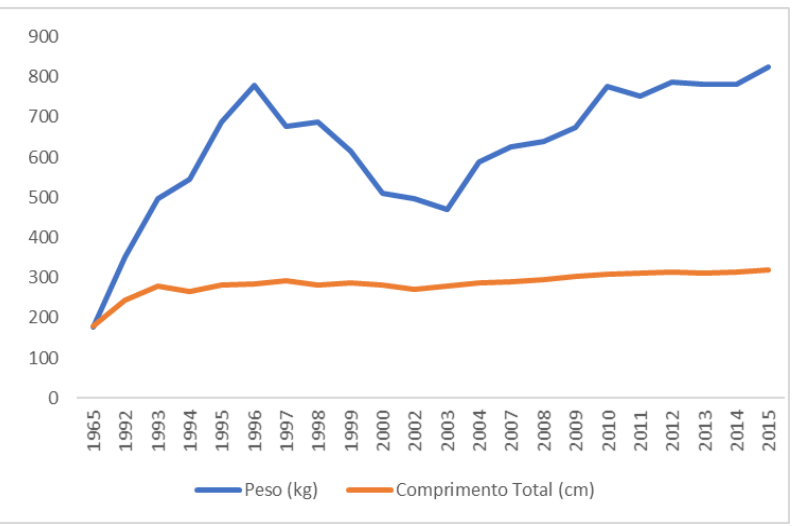

Figura 3. Gráfico de crescimento (Comprimento Total) e evolução (ganho e perda) de peso do peixe-boi marinho Xica, comparando o primeiro manejo realizado em Goiana-PE e em seguida a partir da chegada ao ICMBio/CMA.

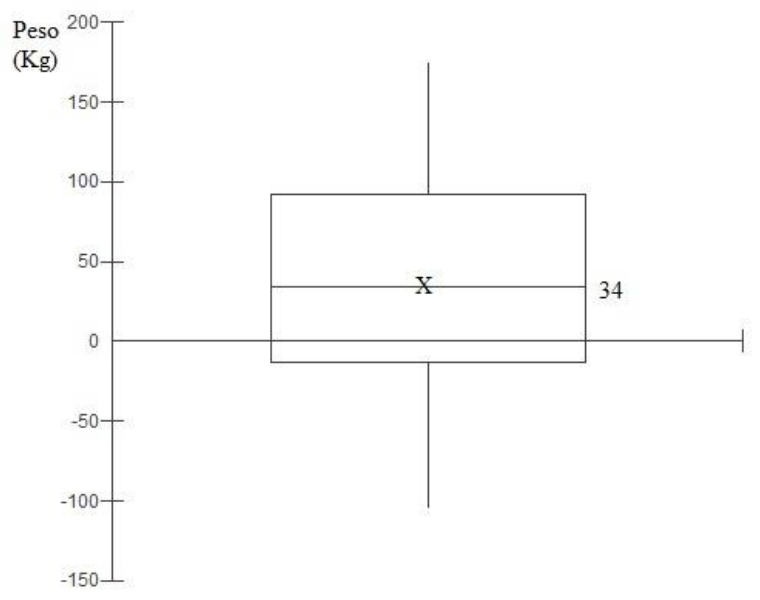

Figura 4. Evolução de peso do peixe-boi marinho Xica no período em que esteve no ICMBio/CMA entre 1992 e 2015.

No período em que a fêmea esteve na praça do Derby, não se tem relato sobre parições. Após a chegada ao ICMBio/CMA, Xica teve quatro parições, sendo dois vivos (dezembro/1996 e junho/2007) e dois natimortos (novembro/2002 e maio/2012).

Em 1996, um filhote denominado Xiquito, nasceu com problemas de conformação óssea e muscular. A causa da deformidade do filhote não foi identificada. A mãe não apresentou cuidados parentais. No dia 05/05/1999 Xiquito veio à óbito, tendo sido o diagnóstico sugestivo de gastroenterite crônica moderada erosiva e esteatose hepática. Não foram realizados exames confirmatórios.

Em 2002, Xica deu à luz a um filhote natimorto. A causa da morte não foi confirmada, mas por ter sido realizada avaliação clínica da fêmea, três meses antes do nascimento, suspeitouse que o peso do corpo da fêmea no útero durante o manejo possa ter prejudicado a saúde do feto. Outra suposição foi de que o feto apresentava problemas genéticos, pois a paternidade foi atribuída a um macho híbrido (T. manatus x $T$. inunguis).

Em 2007, outro filhote vivo, com nome de Chiquinho, nasceu em perfeito estado de saúde. No primeiro mês, a fêmea não apresentou cuidados parentais, além de permanecer com a nadadeira caudal em cima do filhote, dificultando a respiração dele. Por isso, mãe e prole foram mantidas isolados e sob supervisão por dois meses, até a fêmea aceitar o filhote (Figura 5). Com cerca de três anos, Chiquinho foi transferido para recinto de aclimatação e posteriormente solto na natureza, todavia, veio à óbito uma semana após a soltura, por pneumonia.

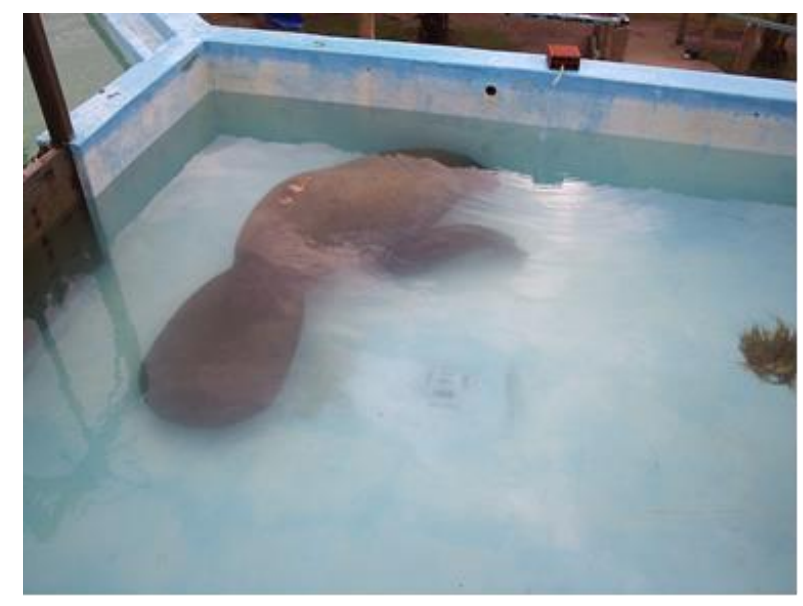

Figura 5. Peixe-bois marinhos Xica e Chiquinho (nascimento em 2007) em processo de treinamento para adaptação dos cuidados parentais.

Em 2012, com 49 anos, Xica teve outra parição de filhote natimorto. A suspeita foi que a idade possa ter acentuado os problemas da escoliose, dificultando que a fêmea pudesse manter a gestação. Buscando salvaguardar a vida de Xica, ela foi separada dos machos, evitando novas gestações.

No dia 14 de junho de 2015 com idade estimada em 52 anos, e detentora do título de peixe-boi-marinho mais velha em cativeiro no Brasil, Xica veio à óbito. Durante a necropsia, verificou-se que a região da coluna apresentava processo inflamatório com doença articular degenerativa crônica, provavelmente causando dor durante grande parte da vida do animal. 
Nos exames histopatológicos foram identificados na área da cicatriz dermatite neutrofílica superficial multifocal, hiperplasia, e fibrose dérmica crônica focal. No sistema musculo esquelético, adjacente à escoliose foi verificado miodegeneração moderada crônica com atrofia de miócitos e ocasional regeneração e hipertrofia. No fígado foi verificado esteatose hepática e no pulmão Congestão moderada. Não foram identificados fatores conclusivos para a morte de Xica, tendo sido esta atribuída as complicações decorrentes da idade e das alterações físicas.

\section{Discussão}

Até 1992, muitos foram os peixes-bois que viveram nas praças do Recife, entretanto Xica foi a mais conhecida (Luna e Attademo, 2020). Estes animais foram mantidos em condições inadequadas, mas vale ressaltar que na época não se tinha o conhecimento da espécie, como se tem nos dias de hoje. O primeiro peixe-boi chegou em exposição no Recife em 1909 e somente em 1980 foi criado o projeto peixe-boi do ICMBio/CMA, onde os estudos foram iniciados (Luna $\mathrm{e}$ Attademo, 2020). Com o aumento do conhecimento e criação de leis de proteção, os recintos inadequados, como o que existia na praça do Derby, deixaram de ser estimulados.

Peixes-bois em cativeiro, comumente apresentam comportamentos estereotipados (Anzolin et al., 2014). As causas destas alterações comportamentais podem ser diversas. No caso de Xica, o comportamento arisco apresentado, pode ser atribuído aos 22 anos em que passou em recinto inadequado e, possivelmente, às dores causadas pela deformação óssea.

As estimativas da massa corporal e crescimento têm sido critérios adotados para a avaliação de desenvolvimento de mamíferos aquáticos (Trites et al, 1998; Borges et al, 2012). O T. manatus atinge uma média de $200 \mathrm{~cm}$ de comprimento total entre 1 e 2 anos de vida e cerca de $300 \mathrm{~cm}$ entre 8-10 anos, podendo atingir até 400cm (Reep e Bonde, 2006; Bonde, 2018; Davis e Walsh, 2018). Em relação ao peso, atingem uma média de até $600 \mathrm{~kg}$, mas podem chegar a pesar 1000kg (Davis \& Walsh, 2018). Xica, ao chegar ao ICMBio/CMA apresentava um peso abaixo da média da espécie $(352 \mathrm{~kg}$ ), mas em dois anos de acompanhamento clínico, fornecimento de dieta adequada e recinto que permitisse seu desenvolvimento, ela atingiu a média esperada (544kg). No óbito, com $825 \mathrm{~kg}$, Xica estava com peso acima da média ( $\square=600 \mathrm{~kg}$ ), mas dentro da margem de peso descrita para a espécie $(<900 \mathrm{~kg})$ (Davis e Walsh, 2018; Luna et al., 2018), demonstrando que os protocolos adotados ao longo dos anos, foram benéficos para o animal.

T. manatus, quando em vida livre, podem consumir uma grande variedade de itens alimentares (algas, capim agulha, folhas de mangue e outros), podendo a chegar a 60 diferentes plantas (Reynold III et al., 2018; Borges et al., 2008). Em cativeiro, nem sempre é possível fornecer estes itens naturais, seja para evitar danos ao meio ambiente ou por inviabilidade de logística para colheita destes itens. Nestas ocasiões, conforme descrito neste trabalho, é importante evitar uma monotonia alimentar, permitindo um equilíbrio nutricional.

Os filhotes vivos de Xica, permitiram a análise da diferença do padrão de crescimento entre filhotes nascidos em cativeiro e resgatados na costa nordeste do Brasil, identificando a necessidade de uma revisão da dieta nutricional dos animais em reabilitação (Borges et al., 2012). Os dois filhotes natimortos, um deles com possibilidade de ter relação com o manejo clínico, alertaram sobre a recomendação de não serem realizados manejos clínicos no seco de peixes-bois fêmeas com suspeita de prenhes. Estes fatores associados resultaram em melhorias no manejo dos animais.

A estimativa de vida do peixe-boi, baseada em estudos de carcaças, vem sendo descrita até 60 anos (Marmontel, 1995; Schwarz e Runge, 2009; Reynolds III et al., 2018), mas esta idade não está bem definida. Um peixe-boi, macho, de nome Snooty, em cativeiro na Flórida viveu até 69 anos (Odell, 2018) e em Dugongos foi registrado um animal de 73 anos (Marsh, 1995). Entretanto, Xica permanece como o peixe-boi-marinho que atingiu a maior idade registrada no Brasil e um dos mais velhos mundialmente.

\section{Considerações Finais}

As condições de cativeiro inadequado até os 22 anos de vida contribuíram para os problemas clínicos e comportamentais apresentados por Xica, sendo, portanto, recomendado que os peixes-bois permaneçam em recintos com espaço suficiente para natação e mergulho. As condutas alimentares e clínicas realizadas durante os anos em que esteve no ICMBio/CMA se demonstraram satisfatórias, permitindo que a fêmea possuísse 
uma grande longevidade. Xica não deixou contribuições de prole, mas demonstrou que fêmeas de peixes-bois, a princípio, podem reproduzir pelo menos até os 49 anos. Em tempo, recomenda-se que fêmeas, com suspeita de prenhes, não sejam manejadas para avaliações clínicas no seco.

\section{Conflito de Interesse}

Os autores declaram não existir conflito de interesse.

\section{Agradecimentos}

Os autores agradecem à toda a equipe do ICMBio/CMA, Projeto Cetáceos da Costa Branca, Fundação Mamíferos Aquáticos e Instituto Mamíferos Aquáticos, que fizeram parte da vida de Xica.

\section{Referências}

Anzolin, D.; Carvalho, P.; Viana, P.; Normande, I.; Souto, A. Stereotypical behaviour in captive West Indian manatee (Trichechus manatus). Journal of the Marine Biological Association of the UK, 94(6): 1133-1137, 2014.

Attademo, F.L.N; Balensiefer, D.C.; Freire, A.C.B.; Souza, G.P.; Cunha, F.A.G.C.; Luna, F.O. Debris ingestion by the Antillean manatee (Trichechus manatus manatus). Marine Pollution Bulletin, 101(1): 284-287, 2015.

Balensiefer, D.C.; Attademo, F.L.N.; Souza, G.P.; Freire, A.C.B.; Cunha, F.A.G.C.; Alencar, A.E.B.; Silva, F.J.L.; Luna, F.O. Three decades of Antillean manatee (Trichechus manatus manatus) strandings along the Brazilian coast. Tropical Conservation Science, 10(1): 1-9, 2017.

Bonde, R.K. Sirenian life history. In: Würsig, B.; Thewissen, J.G.M.; Kovacs, K.M. Encyclopedia of marine mammals. $3^{\text {rd }}$ ed. Massachusetts: Academic Press, 2018. p. 859-861.

Borges, J.C.G.; Araújo, P.G.; Anzolin, D.G.; Miranda, G.E.C. Identificação de itens alimentares constituintes da dieta dos peixesboi marinhos (Trichechus manatus) na região nordeste do Brasil. Biotemas, 21(2): 77-81, 2008.

Borges, J.C.G.; Freire, A.C.B.; Attademo, F.L.N; Lima, I.S.; Anzolin, D.G.; Carvalho, P.S.M.; Vergara-Parente, J.E. Growth pattern differences of captive born Antillean manatee (Trichechus manatus) calves and those rescued in the Brazilian Northeastern coast. Journal of Zoo and Wildlife Medicine, 43(3): 494-500, 2012.

Davis, M.R.; Walsh, M.T. Sirenian medicine. In: Gulland, F.M.D.; Dierauf, L.A.; Whitman, K.L. CRC Handbook of Marine Mammal Medicine. $3^{\text {rd }}$ ed. Boca Raton: Taylor \& Francis, 2018, p.949-968.

Harvey, J.W.; Harr, K.E.; Murphy, D., Walsh, M.T.; Chittick, E.J., Bonde, R.K.; Haubold, E.M. Clinical biochemistry in healthy manatees (Trichechus manatus latirostris). Journal of Zoo and Wildlife Medicine, 38(2), 269-279, 2007.

ICMBio. Livro vermelho da fauna brasileira ameaçada de extinção. Brasília: ICMBio, 2018. v.1, 4162p.

Luna, F.O.; Araújo, J.P.; Lima, R.P.; Pessanha, M.M.; Soavinski, R.J.; Passavante, J.Z.O. Captura e utilização do peixe-boi marinho (Trichechus manatus manatus) no litoral Norte do Brasil. Biotemas, 21(1): 115-123, 2008

Luna, F.O.; Passavante, J.Z.O. Projeto peixeboi/ICMBio. 30 Anos de conservação de uma espécie ameaçada. $1^{\text {a }}$ ed. Brasília: ICMBio, 2010. 108p.

Luna, F.O.; Balensiefer, D.C.; Fragoso, A.B. Stephano, A. Attademo, F.L.N. Trichechus manatus Linnaeus, 1758. In: ICMBio. Livro vermelho da fauna brasileira ameaçada de extinção: Mamíferos. v. 2. Brasília: ICMBio, 2018. p. 103-109.

Luna, F.O.; Attademo, F.L.N., Peixes-bois das praças do Recife - a história de um século de convívio e admiração dos pernambucanos a estes animais. Revista Rural \& Urbano, 5(2): 373-393, 2020.

Marmontel, M. Age and reproduction in female Florida manatees. In: O'Shea, T.J.; Ackerman, B.B.; Percival, H.F. Population biology of the Florida manatee. Washington: National Biological Service Information and Technology Report, 1995. p. 98-119.

Marsh, H. The life history, pattern of breeding, and population dynamics of the dugong. In: O'Shea, T.J.; Ackerman, B.B.; Percival, H.F. Population biology of the Florida manatee. Washington: National Biological Service 
Information and Technology Report, 1995. p. $75-83$.

Mendonça, M.A.; Fonseca, M.S.; Attademo, F.L.N.; Marques, F.S.; Ayres, M.C.C.; Barral, T.D.; Meyer, R.; Portela, R.P. Hematology and clinical biochemistry profiles in Antillean Manatee Trichechus manatus manatus from different types of captivity and free living in Northeast Brazil. Journal of Aquatic Animal Health, 32(4): 168-178, 2020.

Odell, D.K. Sirenian life history. In: Perrin, W.F.; Würsig, B.; Thewissen, J.G.M. Encyclopedia of marine mammals. $3^{\text {rd }}$ ed. San Diego: Academic Press, 2018. p. 10191020.

Reep, R.L.; Bonde, R.K. The Florida manatee: biology and conservation. $1^{\text {st }}$ ed. Gainesville: University Press Florida, 2006, 208p.

Reynolds III, J.E.; Powell, J.A.; Diagne, L.W.K.; Barton, S.L.; Scolardi, K.M. Manatees.
Trichechus manatus, T. senegalensis, and $T$. inunguis. In: Perrin, W.F.; Würsig, B.; Thewissen, J.G.M. Encyclopedia of marine mammals. $3^{\text {rd }}$ ed. San Diego: Academic Press, 2018. p. 558-566.

Rocha, N.B. Memória sobre um exemplar de Trichechus manatus manatus L., 1758 capturado em Goiana (Pernambuco). Arquivos do Museu Nacional do Rio de Janeiro, 54(1): 101-103, 1971.

Schwarz, L.K.; Runge, M.C. Hierarchical bayesian analysis to incorporate age uncertainty in growth curve analysis and estimates of age from length: Florida manatee (Trichechus manatus) carcasses. Canadian Journal of Fisheries and Aquatic Sciences, 66(10): 1775-1789, 2009.

Trites, A.W.; Pauly, D. Estimating mean body masses of marine mammals from maximum body lengths. Canadian Journal of Zoology, 76(5): 886-896, 1998. 